\title{
Feedforward and Feedback Control Performance Assessment for Nonlinear Systems
}

\author{
Zhiguo Wang and Jun Chen \\ Key Laboratory of Advanced Process Control for Light Industry (Ministry of Education), Institute of Automation, \\ Jiangnan University, Wux 214122, China
}

Correspondence should be addressed to Zhiguo Wang; jndx_wzg@aliyun.com

Received 23 January 2014; Revised 29 March 2014; Accepted 30 March 2014; Published 24 April 2014

Academic Editor: Shuping He

Copyright (C) 2014 Z. Wang and J. Chen. This is an open access article distributed under the Creative Commons Attribution License, which permits unrestricted use, distribution, and reproduction in any medium, provided the original work is properly cited.

A performance assessment method for nonlinear feedforward and feedback control systems is proposed in this paper. First, the existence of minimum variance performance bound for two nonlinear systems with different structures is analyzed, and the closedloop model of nonlinear system is obtained with the help of iterative orthogonal least squares identification method. Then, the technology of variance analysis is introduced to establish the variance contributions due to both disturbances and controller. A nonlinear performance index for the feedforward and feedback control systems is estimated using an ANOVA-like variance decomposition method. Finally, a meaningful example is simulated to show the effectiveness of our method.

\section{Introduction}

The technology of control performance assessment (CPA) has attracted much attention in recent years, due to the extensive application of automatic control systems in industrial area. CPA is a management tool to maintain efficient operation performance of automation systems. The main aim is to evaluate the performance of control loops in control systems, diagnose the reason of poor performance, and present effective proposals for improvement once the control performance of a running controller cannot meet the desired requirements.

The study of CPA began to blossom some 20 years ago with the pioneering work by Harris [1]; he proposed a linear performance index based on minimum variance benchmark. Desborough and Harris [2] proposed a normalized performance index for assessment of linear SISO controller performance, which can be estimated by linear regression methods. Stanfelj et al. [3] presented a method that utilized autocorrelation and cross correlation functions for monitoring and diagnosing the cause of poor performance of feedforward and feedback control systems. Desborough and Harris [4] developed a performance assessment algorithm based on variance table to investigate the variance contributions due to disturbances and controllers for a linear feedforward and feedback system. Harris et al. [5] developed a method for assessing the performance of linear MIMO control systems, and this method requires an estimate of the process interactor matrix that characterizes the dead-time structure. Almost at the same time, Huang et al. [6] developed a new approach based on filtering and correlation (FCOR) analysis of the process output and filtered data, which can be used to estimate the controller performance of a general class of linear MIMO processes. Subsequently, Huang et al. [7] developed a method for the performance assessment of linear multivariate feedback plus feedforward control systems using minimum variance control as the benchmark. CPA theoretical issues have been reported by several literatures, such as the references published by Qin [8], Huang and Shah [9], and Jelali [10].

Although the field of CPA has received much attention in theory and engineering in recent years [11-14], the most previous studies are focused on linear systems. In real applications, the industrial processes are naturally nonlinear systems. The estimation of the minimum variance performance lower bound (MVPLB) and the performance index using the linear control performance assessment techniques may be distorted by these nonlinearities. Due to the internal complexity and lack of effective mathematical tools, far less 
has been written on the CPA methods for nonlinear systems. For a special class of nonlinear SISO processes that can be described by the superposition of a nonlinear dynamic model and additive linear disturbance, Harris and Yu [15] presented a method to estimate the MVPLB using closedloop data. Continuing this idea, estimates of the MVPLB for the moderate valve stiction cases are proposed by Yu et al. [16]. Yu et al. [17] proposed a new CPA performance index for general nonlinear SISO models based on an ANOVAlike variance decomposition method. This new performance index is not based on the MVPLB, but it can be used to estimate the MVPLB for some nonlinear systems detailed are discussed in [15]. Considering the process nonlinearity and valve stiction nonlinearity in control system, Zhang [18] proposed some CPA methods for nonlinear systems based on minimum variance benchmark. Yu et al. [19] extended CPA to nonlinear MIMO systems. However, in order to make the problem tractable, they restrict the system structure to be a model with additive linear disturbances and where the nonlinearity is in the form of valve stiction.

In spite of the fact that multivariate control schemes are justified from an economic and quality improvement standpoint, the univariate controllers are the mostly used controllers in practical applications. The performance of these SISO control schemes can be enhanced by including feedforward elements. In this paper, we study the performance assessment for nonlinear feedforward and feedback control systems. The objective of our work is to estimate the MVPLB for this nonlinear system and analyze the contribution of each controller for the overall performance bound. This study has an important guiding significance for the adjustment and design of the actual control system. Two common situations are often encountered in pragmatic feedforward and feedback control systems. The first case is, although a feedforward variable can be measured, it is not used in the control systems; in such situation, the result of CPA for nonlinear feedforward and feedback control systems can provide an estimation of the variance reductions if feedforward controller is considered. In the other case, a feedforward variable is both measured and used in a feedforward and feedback control scheme, and then, the performance of the individual controllers can be assessed by the result of this paper, such that we can determine which controller should be principally adjusted to improve the performance of feedforward and feedback control systems.

Based on some methods for the performance assessment of linear feedforward and feedback control systems, this paper is an extension to nonlinear systems. The outline of this paper is organized as follows. As a prerequisite, the performance assessment of linear feedforward and feedback systems is discussed in Section 2. In Section 3, the existence of MVPLB for nonlinear feedforward and feedback systems is analyzed. In Section 4, a description of the ANOVA-like variance decomposition method is given and a new performance index of nonlinear control systems is proposed. Finally, a simulation is made to illustrate the proposed methodology in Section 5, and this is followed by a conclusion in Section 6.

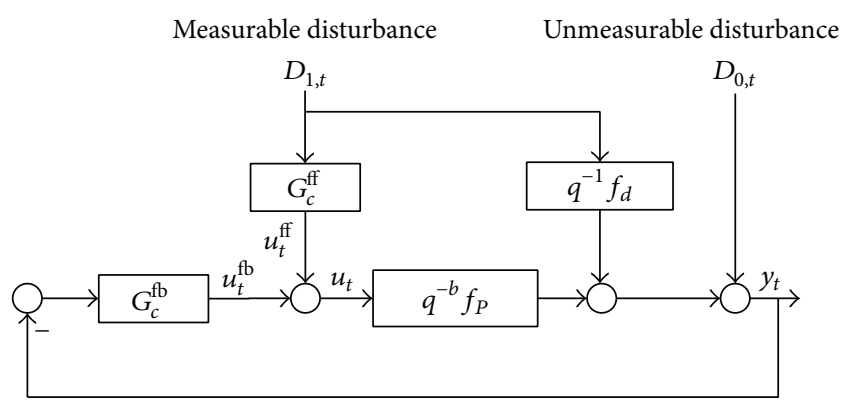

FIgURE 1: Schematic of feedforward and feedback control system.

\section{Analysis of Variance in Linear Feedforward and Feedback Control System}

A structural schematic of general feedforward and feedback control system is given in Figure 1 , where $y_{t}$ is output variable of the process, $u_{t}$ is manipulated variable which is adjusted by summing the outputs from the feedback controller $u_{t}^{\mathrm{fb}}$ and feedforward controller $u_{f}^{\mathrm{ff}}$. $G_{c}^{\mathrm{fb}}$ is feedback controller transfer function, and $G_{c}^{\mathrm{ff}}$ is feedforward controller transfer function. $q^{-b} f_{P}$ represents the process model that may be linear or nonlinear. $b$ is the number of whole periods of process delay. $q^{-l} f_{d} D_{1, t}$ represents the effect that the measured disturbance $D_{1, t}$ has on the process output, and $l$ is the number of periods of delay it takes for a change in $D_{1, t}$ to begin to affect the output. In linear systems, $q^{-l} f_{d} D_{1, t}$ is often expressed by transfer function as $q^{-l} N_{d} D_{1, t}$. $D_{0, t}$ and $D_{1, t}$ represent the unmeasured and measured disturbances, respectively. In this paper, work is based on the assumption that there is no cross correlation among the unmeasured and measured disturbances, and this is reasonable for many industrial processes.

In linear systems, the delay-free process model $f_{P}$ can be represented by the following equation:

$$
f_{P}=\frac{\omega\left(q^{-1}\right)}{\delta\left(q^{-1}\right)},
$$

where $\omega\left(q^{-1}\right)$ and $\delta\left(q^{-1}\right)$ are stable polynomials in the backshift operator $q^{-1}$. Disturbances $D_{0, t}$ and $D_{1, t}$ are represented by autoregressive integrated moving average (ARIMA) time series models:

$$
D_{i, t}=\frac{\theta_{i}\left(q^{-1}\right)}{\varphi_{i}\left(q^{-1}\right) \nabla^{d_{i}}} \alpha_{i, t}, \quad i=0,1
$$

$\left\{\alpha_{i, t}\right\}$ is a sequence of independently and identically distributed random variables with mean zero and constant variance $\sigma_{i}^{2} \cdot \theta_{i}\left(q^{-1}\right)$ and $\varphi_{i}\left(q^{-1}\right)$ are monic and stable polynomials. The difference operator is defined as $\nabla \stackrel{\text { def }}{=}\left(1-q^{-1}\right)$, and $d_{i}$ is the degree of differencing. The linear feedforward 
and feedback control system can be modeled as the sum of two disturbances and a linear transfer function:

$$
y_{t}=q^{-b} \frac{\omega\left(q^{-1}\right)}{\delta\left(q^{-1}\right)} u_{t}+D_{0, t}+q^{-l} N_{d} D_{1, t}
$$

Substituting the feedforward and feedback controller representation into above equation and multiplying both sides by $q^{d}$ and collecting terms

$$
y_{t+b}=\frac{\omega\left(q^{-1}\right)}{\delta\left(q^{-1}\right)} u_{t}^{\mathrm{fb}}+D_{0, t+b}+\frac{\omega\left(q^{-1}\right)}{\delta\left(q^{-1}\right)} u_{t}^{\mathrm{ff}}+q^{-l} N_{d} D_{1, t+b} .
$$

In an analogous manner to the minimum variance feedback controller, the design of minimum variance feedforward and feedback controller can be derived. The research result of Desborough and Harris [4] reported that the linear closedloop system can be described in terms of the unmeasured disturbance driving force and the measured feedforward variable. We do the similar work, which yields

$$
\begin{aligned}
y_{t+b}= & \frac{\omega\left(q^{-1}\right)}{\delta\left(q^{-1}\right)}\left(-G_{c}^{\mathrm{fb}} y_{t}\right)+\frac{\theta_{0}\left(q^{-1}\right)}{\varphi_{0}\left(q^{-1}\right) \nabla^{d_{0}}} \alpha_{0, t+b} \\
& +\frac{\omega\left(q^{-1}\right)}{\delta\left(q^{-1}\right)} G_{c}^{\mathrm{ff}} D_{1, t}+q^{-l} N_{d} D_{1, t+b} \\
= & \frac{\theta_{0}\left(q^{-1}\right) / \varphi_{0}\left(q^{-1}\right) \nabla^{d_{0}}}{1+q^{-b}\left[\omega\left(q^{-1}\right) / \delta\left(q^{-1}\right)\right] G_{c}^{\mathrm{fb}}} \alpha_{0, t+b} \\
& +\frac{q^{-b} G_{P}\left(q^{-1}\right) G_{c}^{\mathrm{ff}}+q^{-l} N_{d}}{1+q^{-b}\left[\omega\left(q^{-1}\right) / \delta\left(q^{-1}\right)\right] G_{c}^{\mathrm{fb}}} D_{1, t+b} \\
= & \psi_{0}\left(q^{-1}\right) \alpha_{0, t+b}+\psi_{1}\left(q^{-1}\right) D_{1, t+b},
\end{aligned}
$$

where $\psi_{0}\left(q^{-1}\right)$ is the closed-loop transfer function between $y_{t}$ and the driving force for the unmeasured disturbance. $\psi_{1}\left(q^{-1}\right)$ is the closed-loop transfer function between $y_{t}$ and measured feedforward variable $D_{1, t}$. Alternatively, the process can be described in terms of the driving forces alone:

$$
y_{t}=\psi_{0}\left(q^{-1}\right) \alpha_{0, t+b}+\psi_{1}\left(q^{-1}\right) \alpha_{1, t+b} .
$$

Each of the closed-loop transfer functions in (6) can be expanded in a convergent power series in $q^{-1}$ :

$$
\psi_{i}\left(q^{-1}\right)=\sum_{h=0}^{\infty} \psi_{i, h} q^{-h}
$$

This expansion is obtained by writing each transfer function as a ratio of polynomials $q^{-1}$ and then dividing the numerator into the denominator using polynomial long division. Then the process output can be extended as

$$
y_{t+b}=y_{0, t+b}+y_{1, t+b}=\sum_{h=0}^{\infty} \psi_{0, h} q^{-h} \alpha_{0, t+b}+\sum_{h=0}^{\infty} \psi_{1, h} q^{-h} \alpha_{1, t+b} .
$$

The term $y_{0, t+b}$ is the contribution of unmeasured disturbance $D_{0, t}$ to the process output; it can be written as

$$
\begin{aligned}
y_{0, t+b}= & \left(1+\psi_{0,1} q^{-1}+\cdots+\psi_{0, b-1} q^{-(b-1)}\right) \alpha_{0, t+b} \\
& +\left(\psi_{0, b} q^{-b}+\psi_{0, b+1} q^{-(b+1)}+\cdots\right) \alpha_{1, t+b} \\
= & e_{0, t+b / t}+\sum_{h=b}^{\infty} \psi_{0, h} q^{-h} \alpha_{0, t+b}=e_{0, t+b / t}+y_{0, t+b}^{\mathrm{fb}} .
\end{aligned}
$$

The first term $e_{0, t+b / t}$ in above function is recognized as the prediction error, which is independent of the second term. The second term is the contribution to the process output $y_{0, t+b}$ which arises from the nonoptimality of the control associated with the unmeasured disturbance, and it is also a function of the process dynamics, the unmeasured disturbance, and the feedback controller only.

In a similar manner, the contribution of the measured disturbance $D_{1, t}$ to the process output can be written as

$$
\begin{aligned}
y_{1, t+b}= & e_{1, t+b / t}+\left(\psi_{1, b} q^{-b}+\cdots+\psi_{1, b+l-1} q^{-(b+l-1)}\right) \alpha_{1, t+b} \\
& +\left(\psi_{1, b+l} q^{-(b+l)}+\psi_{1, b+l+1} q^{-(b+l+1)}+\cdots\right) \alpha_{1, t+b} \\
= & e_{1, t+b / t}+\sum_{h=b}^{b+l-1} \psi_{1, h} q^{-h} \alpha_{1, t+b} \\
& +\sum_{h=b+l}^{\infty} \psi_{1, h} q^{-h} \alpha_{1, t+b}=e_{1, t+b / t}+y_{1, t+b}^{\mathrm{ff}}+y_{1, t+b}^{\mathrm{ff} \& \mathrm{fb}}
\end{aligned}
$$

where

$$
\begin{aligned}
& e_{1, t+b / t} \\
& = \begin{cases}0, & l \geq b \\
(\underbrace{\psi_{1,0} q^{0}+\cdots+\psi_{1, l-1} q^{-(l-1)}}_{0}+\psi_{1, l} q^{-l} & \\
\left.+\psi_{1, l+1} q^{-(l+1)}+\cdots+\psi_{1, b-1} q^{-(b-1)}\right) \alpha_{1, t+b} & l<b .\end{cases}
\end{aligned}
$$

In (10), the first term $e_{1, t+b / t}$ is the prediction error for the measured disturbance, and it is independent of the second and third terms. The second term is the contribution to the output $y_{1, t+b}$ which arises from the nonoptimality of the feedforward controller only, and the third term is the contribution which arises from the combined effect of the nonoptimality of the feedforward controller and the feedback controller.

Since it has been assumed that the measured and unmeasured disturbances are not cross correlated, the prediction error $e_{0, t+b / t}$ and $e_{1, t+b-l / t}$ are independent of all the controllers. Then, the process output under minimum variance control is given by the sum of the individual error in forecasting the effect of the disturbances:

$$
y_{t+b}^{\mathrm{mv}}=e_{0, t+b / t}+e_{1, t+b-l / t},
$$

and the MVPLB can be written as

$$
\sigma_{\mathrm{mv}}^{2}=\operatorname{var}\left(e_{0, t+b / t}\right)+\operatorname{var}\left(e_{1, t+b / t}\right) .
$$




\section{MVPLB of Nonlinear Feedforward and Feedback Control System}

Due to the effect of various factors such as complexity of nonlinear behavior and challenges in model determination and parameter estimation, far less has been written to extend the methods for performance assessment to nonlinear systems. In order to simplify the analysis and without loss of generality, the problem of estimation for minimum variance performance bound for nonlinear feedforward and feedback systems is given in two aspects.

First, we only assume that the process model has a nonlinear representation in the structural schematic Figure 1, and this is not very restrictive in many applications. Then, the closed output $b$-steps into the future of the nonlinear system can be expressed as

$$
y_{t+b}=f_{P}\left(u_{t}^{*}\right)+D_{0, t+b}+N_{d} D_{1, t+b-l} \text {, }
$$

where the notation $f_{P}(\cdot)$ denotes a nonlinear function of process model, and the superscript $*$ is used to represent the vector collecting the immediate historical values; that is, $u_{t}^{*} \stackrel{\text { def }}{=}\left(u_{t-1}, \ldots, u_{t-n_{u}}\right)$. Decomposing the unmeasured disturbance $D_{0, t+b}$ into a prediction error and a prediction

$$
D_{0, t+b}=e_{0, t+b / t}+\widehat{D}_{0, t+b / t},
$$

the prediction $\widehat{D}_{0, t+b / t}$ is the $b$-step ahead minimum mean square error prediction for the value of the unmeasured disturbance $b$ steps into the future. The effects of the measured feedforward variables are also decomposed into a prediction error and a prediction

$$
N_{d} D_{1, t+b-l}=e_{1, t+b-l / t}+\widehat{D}_{1, t+b-l / t} .
$$

The prediction $\widehat{D}_{1, t+b-l / t}$ is the $b-l$ step ahead minimum mean square error prediction for the value of the measured disturbance $b-l$ steps into the future. Note that if $l$ is greater than or equal to $b$, then $e_{1, t+b-l / t}=0$. This implies that there is no prediction error since we exactly know the future value of the effect on the process of the measured disturbance.

The minimum variance control law is found by minimizing the mean square error of the output:

$$
\begin{aligned}
y_{t+b} & =f_{P}\left(u_{t}^{*}\right)+D_{0, t+b}+N_{d} D_{1, t+b-l} \\
& =f_{P}\left(u_{t}^{*}\right)+e_{0, t+b / t}+\widehat{D}_{0, t+b / t}+e_{1, t+b-l / t}+\widehat{D}_{1, t+b-l / t} \\
& =\underbrace{e_{0, t+b / t}+e_{1, t+b-l / t}}_{\text {term } 1}+\underbrace{f_{P}\left(u_{t}^{*}\right)+\widehat{D}_{0, t+b / t}+\widehat{D}_{1, t+b-l / t}}_{\text {term } 2} .
\end{aligned}
$$

It follows from this formula that the minimum variance controller (MVC) set the manipulated variables to exactly cancel the predictions; that is,

$$
f_{P}\left(u_{t}^{\mathrm{fb}}, u_{t}^{\mathrm{ff}}\right)+\widehat{D}_{0, t+b / t}+\widehat{D}_{1, t+b-l / t}=0
$$

Then the process output under this control scheme can be denoted by

$$
y_{t+b}^{\mathrm{mv}}=e_{0, t+b / t}+ \begin{cases}0, & l \geq b \\ e_{1, t+b-l / t}, & l<b .\end{cases}
$$

As we have assumed that there is no cross correlation among the unmeasured and measured disturbances, the prediction errors $e_{0, t+b / t}$ and $e_{1, t+b-l / t}$ are independent and unrelated with controller parameters. Then, the MVPLB of closed-loop output is

$$
\begin{aligned}
\sigma_{\mathrm{mv}}^{2}= & \operatorname{var}\left(e_{0, t+b / t}\right)+\operatorname{var}\left(e_{1, t+b-l / t}\right) \\
= & \left(1+\psi_{0,1}^{\prime 2}+\cdots+\psi_{0, b-1}^{\prime 2}\right) \sigma_{0}^{2} \\
& + \begin{cases}0 & l \geq b \\
\left(\psi_{1,0}^{\prime 2}+\psi_{1,1}^{\prime 2}+\cdots+\psi_{1, b-l-1}^{\prime 2}\right) \sigma_{1}^{2} & l<b .\end{cases}
\end{aligned}
$$

From above derivation, we can conclude that the MVPLB of nonlinear feedforward and feedback system is identical to that of linear system. The difference is that it is possible to adopt different controllers for obtaining same minimum variance.

Second, a more general form of nonlinear feedforward and feedback control systems is considered:

$$
y_{t}=\underbrace{q^{-b} f_{P}\left(u_{t}^{*}\right)}_{\text {nonlinear }}+\underbrace{\widetilde{D}_{0, t}}_{\text {nonlinear }}+\underbrace{\widetilde{D}_{1, t-l}}_{\text {nonlinear }},
$$

where the terms $\widetilde{D}_{0, t}$ and $\widetilde{D}_{1, t-l}$ are called output disturbances which represent the effect that the unmeasured and measured disturbances have on the process output, respectively. They are also nonlinear and can be represented by nonlinear ARMA model as

$$
\begin{aligned}
& \widetilde{D}_{0, t}=f_{0, D}\left(\widetilde{D}_{0, t-1}^{*}, \alpha_{0, t-1}^{*}\right)+\alpha_{0, t}, \\
& \widetilde{D}_{1, t}=f_{d} D_{1, t}=f_{1, D}\left(\widetilde{D}_{1, t-1}^{*}, \alpha_{1, t-1}^{*}\right)+\alpha_{1, t} .
\end{aligned}
$$

Further, we assume that the output disturbance admits a representation of the form

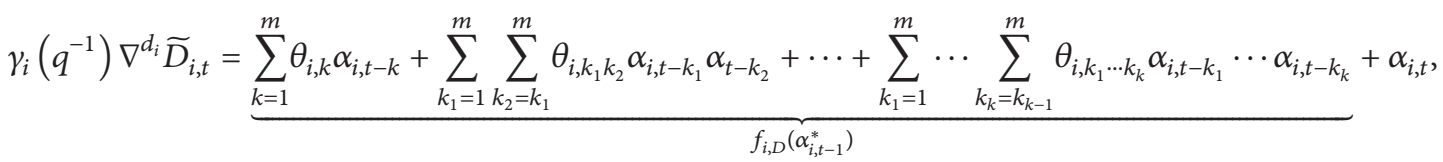


where $\left\{\alpha_{i, t}\right\}$ is a white noise sequence with mean $\mu_{i, \alpha}$ and variance $\sigma_{i, \alpha}^{2}$, and $\gamma_{i}\left(q^{-1}\right)$ is monic and stable polynomial, and we also assume that the disturbance model is invertible. Multiply both sides by $q^{b}$ and substitute for all values of $y_{t+b-i}$, $i=1, \ldots, b-1$, in $(21)$ :

$$
\begin{aligned}
y_{t+b}= & f_{P}\left(u_{t}^{*}\right)+\sum_{j}^{b-1} \tau_{0, j}\left(f_{0, D}\left(\alpha_{0, t+b-1-j}^{*}\right)+\alpha_{0, t+b-j}\right) \\
& +K_{0, b}\left(\widetilde{D}_{0, t}, \alpha_{0, t}^{*}\right) \\
& +\sum_{j}^{b-l-1} \tau_{1, j}\left(f_{1, D}\left(\alpha_{1, t+b-l-1-j}^{*}\right)+\alpha_{1, t+b-l-j}\right) \\
& +K_{1, b}\left(\widetilde{D}_{1, t}, \alpha_{1, t}^{*}\right),
\end{aligned}
$$

where $\tau_{i, j}$ is the $j$ th impulse coefficient of $\left[\gamma_{i}\left(q^{-1}\right) \nabla^{d_{i}}\right]^{-1}, i=$ 0 or $1 . K_{i, b}\left(\widetilde{D}_{i, t}, \alpha_{i, t}^{*}\right)$ is a remainder term that is obtained by successive substitutions. The unmeasured output disturbance is represented as

$$
\begin{aligned}
\widetilde{D}_{0, t+b}= & \sum_{j}^{b-1} \tau_{0, j}\left(f_{0, D}\left(\alpha_{0, t+b-1-j}^{*}\right)+\alpha_{0, t+b-j}\right) \\
& +K_{0, b}\left(\widetilde{D}_{0, t}, \alpha_{0, t}^{*}\right) .
\end{aligned}
$$

According to the definition of conditional expectation, the $b$ step ahead prediction is

$$
\begin{aligned}
\widehat{\widetilde{D}}_{0, t+b / t}= & E\left\{\sum_{j}^{b-1} \tau_{0, j}\left(f_{0, D}\left(\alpha_{0, t+b-1-j}^{*}\right)+\alpha_{0, t+b-j}\right) \mid I_{t}\right\} \\
& +E\left\{K_{0, b}\left(\widetilde{D}_{0, t}, \alpha_{0, t}^{*}\right)\right\} \\
= & E\left\{\sum_{j}^{b-1} \tau_{0, j}\left(f_{0, D}\left(\alpha_{0, t+b-1-j}^{*}\right)+\alpha_{0, t+b-j}\right) \mid I_{t}\right\} \\
& +K_{0, b}\left(\widetilde{D}_{0, t}, \alpha_{0, t}^{*}\right) .
\end{aligned}
$$

Now in the aforementioned equation, we know

$$
\begin{aligned}
& E\left\{\alpha_{0, t+k} \mid I_{t}\right\}=\mu_{0, \alpha}, \quad k=1, \ldots . b, \\
& E\left\{\alpha_{0, t-k} \mid I_{t}\right\}=\alpha_{0, t-k}=\widetilde{D}_{0, t-k}-\widehat{\widetilde{D}}_{0, t-k / t-k-1}, \quad k \geq 0, \\
& E\left\{f_{0, D}\left(\alpha_{0, t+k}^{*}\right) \mid I_{t}\right\} \\
& =\int_{-\infty}^{\infty} \cdots \int_{-\infty}^{\infty} f_{0, D}\left(\alpha_{0, t+k}^{*}\right) \\
& \quad \times p_{0}\left(\alpha_{0, t+k}, \ldots, \alpha_{0, t+1}\right) d \alpha_{0, t+k} \cdots d \alpha_{0, t+1},
\end{aligned}
$$

where $p_{0}\left(\alpha_{0, t+k}, \ldots, \alpha_{0, t+1}\right)$ is the joint distribution of $\alpha_{0, t+k} \cdots \alpha_{0, t+1}$. Then the prediction error for the unmeasured output disturbance is

$$
\begin{aligned}
\widetilde{e}_{0, t+b / t}= & \widetilde{D}_{0, t+b}-\widehat{\widetilde{D}}_{0, t+b / t} \\
=\sum_{j=0}^{b-1} \tau_{0, j}( & f_{0, D}\left(\alpha_{0, t+b-1-j}^{*}\right)-E\left\{f_{0, D}\left(\alpha_{0, t+b-1-j}^{*}\right) \mid I_{t}\right\} \\
& \left.+\alpha_{0, t+b-j}-\mu_{0, \alpha}\right) .
\end{aligned}
$$

In a same manner, the prediction error for the measured output disturbance is

$$
\begin{aligned}
& \widetilde{e}_{1, t+b-l / t}=\widetilde{D}_{1, t+b-l}-\widehat{\widetilde{D}}_{1, t+b-l / t} \\
& = \begin{cases}0, & l \geq b \\
\sum_{j=0}^{b-l-1} \tau_{1, j}\left(f_{1, D}\left(\alpha_{1, t+b-l-1-j}^{*}\right)\right. & \\
-E\left\{f_{1, D}\left(\alpha_{1, t+b-l-1-j}^{*}\right) \mid I_{t}\right\} & \\
\left.+\alpha_{1, t+b-l-j}-\mu_{1, \alpha}\right), & l<b .\end{cases}
\end{aligned}
$$

The process output can be written as

$$
y_{t+b}=f_{P}\left(u_{t}^{*}\right)+\widehat{\widetilde{D}}_{0, t+b / t}+\widehat{\widetilde{D}}_{1, t+b-l / t}+\widetilde{e}_{0, t+b / t}+\widetilde{e}_{1, t+b-l / t} .
$$

If it is possible to find the control action at time $t$ such that

$$
f_{P}\left(u_{t}^{*}\right)+\widehat{\widetilde{D}}_{0, t+b / t}+\widehat{\widetilde{D}}_{1, t+b-l / t}=0,
$$

then the resulting controller is the minimum variance controller. It may not be possible to implement a minimum variance controller due to the various reasons. For instance, it may lead to excessive manipulated variable action and may not be robust to modeling errors. However, the output variance set by minimum variance provides a theoretical lower bound on the system output and can be used as a useful guide for controller assessment.

The process output under minimum variance control is given by the sum of the individual error in predicting the effect of the disturbances:

$$
y_{t+b}^{\mathrm{mv}}=\widetilde{e}_{0, t+b / t}+ \begin{cases}0, & l \geq b \\ \widetilde{e}_{1, t+b-l / t}, & l<b .\end{cases}
$$

It should be pointed out that the terms $\widetilde{e}_{0, t+b / t}$ and $\widetilde{e}_{1, t+b-l / t}$ are very complicated functions, and they may not be expanded in convergent time series as that in linear systems. Therefore, it is difficult to estimate the MVPLB from the closed-loop operation data of feedforward and feedback control system by using traditional linear regression method. But we can get a conclusion that the MVPLB does not depend on the manipulated variable and only related with the most recent $b$ past unmeasured disturbance driving force and $b-l$ past measured disturbance driving force. 


\section{ANOVA-Based Performance Assessment of Nonlinear Feedforward and Feedback Control System}

Analysis of variance (ANOVA) methods are a class of statistical methods that are useful in process systems engineering. Its primary task is to decompose the variance of a response variable into contributions arising from the inputs and assess the magnitude and significance of each of their contributions. Historically, the ANOVA variance decomposition techniques were used to provide variance analysis for nonlinear systems with the multidisturbance sources [20].

For the output of a static system such as $Y=$ $f\left(X_{1}, X_{2}, \ldots, X_{P}\right)$, the relative importance of the independent inputs can be quantified by the fractional variance, and this can be calculated using an ANOVA-like decomposition formula [21]:

$$
\operatorname{Var}[Y]=\sum_{i} V_{i}+\sum_{i} \sum_{j>i} V_{i j}+\cdots+V_{12 \cdots p},
$$

where $V_{i}=\operatorname{Var}\left[E\left[Y \mid X_{i}=x_{i}\right]\right], V_{i}=\operatorname{Var}\left[E\left[Y \mid X_{i}=x_{i}\right]\right]$, $V_{i j}=\operatorname{Var}\left[E\left[Y \mid X_{i}=x_{i}, X_{j}=x_{j}\right]\right]-\operatorname{Var}\left[E\left[Y \mid X_{i}=\right.\right.$ $\left.\left.x_{i}\right]\right]-\operatorname{Var}\left[E\left[Y \mid X_{j}=x_{j}\right]\right]$ and so on. $E\left[Y \mid X_{i}=x_{i}\right]$ denotes the expectation of $Y$ conditional on $X_{i}$ when fixing the value $x_{i}$, and $V$ stands for variance over all the possible values of $x_{i}$. In the same way, if we partition the variable set $\left(X_{1}, X_{2}, \ldots, X_{P}\right)$ into two groups: $U_{1}=\left(X_{1}, \ldots, X_{k}\right)$ and $U_{2}=\left(X_{P-k+1}, \ldots, X_{P}\right)$, then the variance of $Y=f\left(U_{1}, U_{2}\right)$ can be decomposed into $V[Y]=V_{U_{1}}+V_{U_{2}}+V_{U_{1} U_{2}}$.

For the nonlinear feedforward and feedback control systems described by Figure 1, we separate the disturbance entering the system after time 0 , say $\left[\alpha_{0, t+b}, \alpha_{0, t+b-1}, \ldots\right.$, $\left.\alpha_{0,1}, \alpha_{1, t+b-l}, \alpha_{1, t+b-l-1}, \ldots, \alpha_{1,1}\right]$, into two groups: $x_{1}=$ $\left[\alpha_{0, t+b}, \ldots, \alpha_{0, t+1}, \alpha_{1, t+b-l}, \ldots, \alpha_{1, t+1}\right]$ and $x_{2}=\left[\alpha_{0, t}, \ldots\right.$, $\left.\alpha_{0,1}, \alpha_{1, t}, \ldots, \alpha_{1,1}\right]$. The first group includes all the disturbances entering the system after time $t$ and the second group includes all the disturbances entering the system up to and including time $t$ and including time $t$ starting from the initial time $t=0$. Now, we are interested in determining the sensitivity of output $y_{t+b}$ variations of two vector series $x_{1}$ and $x_{2}$. Since the future behavior of $y_{t+b}$ is possibly dependent on initial conditions due to the nonlinearity, the initial condition must be considered before using the ANOVA-like decomposition equation. Using the well-known variance decomposition theorem, the variance of $y_{t+b}$ can be decomposed into two terms:

$$
V\left[y_{t+b}\right]=E_{I_{0}}\left[V_{x}\left[y_{t+b} \mid I_{0}\right]\right]+V_{I_{0}}\left[E_{x}\left[y_{t+b} \mid I_{0}\right]\right],
$$

where $x=\left[x_{1}, x_{2}\right]$ denotes all of disturbances entering the system from time 1 to time $t+b$ and $I_{0}$ denotes initial conditions. The first term in above equation is the fractional contribution to the variance of $y_{t+b}$ from the disturbance signal and the interaction between disturbance and the initial condition. The second term is the fractional contribution to the output solely due to the uncertainties in the initial condition. Given the initial condition $I_{0}$, conditional variance $V_{x}\left[y_{t+b} \mid I_{0}\right]$ can be decomposed as

$$
V_{x}\left|I_{0}=V_{x}\left[y_{t+b} \mid I_{0}\right]=V_{1}\right| I_{0}+V_{2}\left|I_{0}+V_{12}\right| I_{0},
$$

where $V_{1}\left|I_{0}=V_{x 1}\left[E_{x 2}\left[y_{t+b} \mid\left(x_{1}, I_{0}\right)\right]\right], V_{2}\right| I_{0}=$ $V_{x 2}\left[E_{x 1}\left[y_{t+b} \mid\left(x_{2}, I_{0}\right)\right]\right]$, and $V_{12} \mid I_{0}=V_{x}\left[E_{x}\left[y_{t+b} \mid\right.\right.$ $\left.\left.\left(x, I_{0}\right)\right]\right]-V_{1}\left|I_{0}-V_{2}\right| I_{0} . E_{I_{0}}\left[V_{1} \mid I_{0}\right]$ denotes the main effect of $x_{1}$ on the $V\left[y_{t+b}\right] . E_{I_{0}}\left[V_{2} \mid I_{0}\right]$ denotes the interaction contributing to the $V\left[y_{t+b}\right]$ that is not accounted for the main effects of $x_{1}$ and $x_{2}$. Consequently, a suitable performance index can be constructed by referring to Harris index:

$$
\eta_{t}=\frac{E_{I_{0}}\left[V_{1} \mid I_{0}\right]}{\operatorname{Var}\left[y_{t+b}\right]} .
$$

If the nonlinear model is stationary, then the distribution of $\lim _{t \rightarrow \infty} y_{t+b}$ can reach an equilibrium. For linear time series, this limiting distribution is independent of initial condition. But for a stationary nonlinear model, the limiting distribution may depend on the initial condition. Therefore, the performance index $\eta_{t}$ will depend on the initial condition. If the distribution of $\lim _{t \rightarrow \infty} y_{t+b}$ does not depend on the initial conditions, the process is termed ergodic. In actual industry, the cases that processes are strongly nonergodic are more pathological than common cases. For an ergodic nonlinear system, $V_{I_{0}}\left[E_{x}\left[Y_{t+b} \mid I_{0}\right]\right]$ in (34) will be zero for $t \rightarrow \infty$, and the variance decomposition can be expressed when $t \rightarrow \infty$ as

$\operatorname{Var}\left[y_{t+b}\right]=E_{I_{0}}\left[V_{1}\left|I_{0}+V_{2}\right| I_{0}+V_{12} \mid I_{0}\right]=V_{1}+V_{2}+V_{12}$,

where $V_{1}=V_{x 1}\left[E_{x 2}\left[y_{t+b} \mid x_{1}\right]\right], V_{2}=V_{x 2}\left[E_{x 1}\left[y_{t+b} \mid x_{2}\right]\right]$, and $V_{12}=V\left[y_{t+b}\right]-V_{1}-V_{2}$. The performance index will turn into

$$
\lim _{t \rightarrow \infty} \eta_{t}=\lim _{t \rightarrow \infty} \frac{V_{1}}{\operatorname{Var}\left[y_{t+b}\right]} .
$$

Generally, we will approximate the infinite limit in above equation by some suitably large value $\eta_{M}$.

In Section 3, we conclude that the MVPLB of nonlinear feedforward and feedback control systems is existent and only related with the most recent $b$ past unmeasured disturbance driving force and $b-l$ past measured disturbance driving force. Moreover, we have $x_{1}=$ $\left[\alpha_{0, t+b}, \ldots, \alpha_{0, t+1}, \alpha_{1, t+b-l}, \ldots, \alpha_{1, t+1}\right]$, so $\eta_{t}$ just is the minimum variance performance index of the nonlinear feedforward and feedback control systems.

For the computation of the performance index, the principal task is to estimate the closed-loop model of nonlinear feedforward and feedback control system. Firstly, the measured feedforward variable transfer function, given in (2), must be estimated. Using the linear regression techniques and past values of $D_{1, t}$. The model of measured disturbance can be estimated by

$$
D_{1, t}=\sum_{i=1}^{J_{D}} \lambda_{i} D_{1, t-i}+\widehat{\alpha}_{1, t} .
$$

$\widehat{\alpha}_{1, t}$ is an estimate of the independent driving force for measured disturbance. If the process is controlled by a linear or nonlinear feedforward and feedback controller such as 
$u_{t}=g\left(y_{t}, \ldots, y_{t-n_{y}}\right)$, then the output of closed-loop system can be written as

$$
\begin{aligned}
y_{t+b} & \\
& =f_{1}\left(y_{t}, \ldots, y_{t-n_{y}}, \alpha_{0, t+b}, \ldots, \alpha_{0, t-n_{0}}, D_{1, t+b-l}, \ldots, D_{1, t-n_{D}}\right) \\
& =f_{2}\left(y_{t}, \ldots, y_{t-n_{y}}, \alpha_{0, t+b}, \ldots, \alpha_{0, t-n_{0}}, \widehat{\alpha}_{1, t+b-l}, \ldots, \widehat{\alpha}_{1, t-n_{1}}\right) .
\end{aligned}
$$

According to the existing knowledge, any continuous $f(\cdot)$ can be arbitrarily well approximated by polynomial models. Therefore, expanding $f_{2}(\cdot)$ in above equation as a polynomial of degree $l$ gives the representation

$$
\begin{aligned}
y_{t+b}= & \varepsilon_{0}+\sum_{i_{1}=1}^{n} \varepsilon_{i_{1}} x_{i_{1}, t}+\sum_{i_{1}=1}^{n} \sum_{i_{2}=i_{1}}^{n} \varepsilon_{i_{1} i_{2}} x_{i_{1}, t} x_{i_{2}, t}+\cdots \\
& +\sum_{i_{1}=1}^{n} \cdots \sum_{i_{l}=i_{l-1}}^{n} \varepsilon_{i_{1} \cdots i_{l}} x_{i_{1}, t} \cdots x_{i_{l}, t}+\xi_{t},
\end{aligned}
$$

where

$$
n=n_{y}+n_{0}+n_{1}
$$

and $x_{1, t}=y_{t}, x_{2, t}=y_{t-1}, \ldots, x_{n_{y}, t}=y_{t-n_{y}}, x_{n_{y}+1, t}=\alpha_{0, t+b}, \ldots$, $x_{n_{y}+n_{0}, t}=\alpha_{0, t-n_{0}}$, and $x_{n_{y}+n_{0}+1, t}=\alpha_{1, t+b-l}, \ldots, x_{n, t}=\alpha_{1, t-n_{1}}$. Moreover, the output of closed-loop system can be written as a linear regression model:

$$
y_{t+b}=\sum_{i=1}^{M} p_{i, t} \varepsilon_{i}+\xi_{t}, \quad t=1, \ldots, N
$$

where $N$ is the data length, the $p_{i, t}$ are monomials of $x_{1, t}$ to $x_{n, t}$ up to degree $l, p_{1, t}=1$ corresponding to a constant term, $\xi_{t}$ is some modeling error, and the $\varepsilon_{i}, i=1, \ldots, M$, are unknown parameters to be estimated. Then above equation can be written in the matrix form

$$
\mathbf{Y}=\mathbf{P} \Theta+\mathbf{E}
$$

where

$$
\begin{array}{ll}
\mathbf{Y}=\left[\begin{array}{c}
y_{1} \\
\vdots \\
y_{N}
\end{array}\right], \quad \mathbf{P}=\left[\begin{array}{c}
p_{1} \\
\vdots \\
p_{M}
\end{array}\right]^{T}=\left[\begin{array}{ccc}
p_{1,1} & \cdots & p_{M, 1} \\
\vdots & \ddots & \vdots \\
p_{1, N} & \cdots & p_{M, N}
\end{array}\right], \\
\boldsymbol{\Theta}=\left[\begin{array}{c}
\varepsilon_{1} \\
\vdots \\
\varepsilon_{M}
\end{array}\right], \quad \mathbf{E}=\left[\begin{array}{c}
\xi_{1} \\
\vdots \\
\xi_{N}
\end{array}\right] .
\end{array}
$$

In reality, as parameters $n_{y}, n_{0}$, and $n_{1}$ are unknown, we must consider the combined problem of structure selection and parameter estimation. To avoid losing significant terms which must be included in the final model, we are forced to consider the full model set at the beginning of the identification and then to select a subset from full model set and find the corresponding parameter. The orthogonal least squares
(OLS) method [22] can be used to determine the order and estimate the parameters of the model. Denote

$$
\widetilde{P}^{(0)}=[P: Y] \text {. }
$$

After a series of Householder transformations $H^{(i)}, i=$ $1, \ldots, k-1$ have been successively applied to $\widetilde{P}^{(0)}$; it is transformed to

$$
\widetilde{P}^{(k-1)}=\left[\begin{array}{lllll}
\widetilde{R}_{k-1} & \tilde{p}_{k}^{(k-1)} & \ldots & \widetilde{p}_{M}^{(k-1)}: & Y^{(K-1)}
\end{array}\right],
$$

where $\widetilde{R}_{k-1}=\left(\begin{array}{ll}R_{k-1} & 0\end{array}\right)^{T}, \widetilde{p}_{k}^{(k-1)}=\left(\widetilde{p}_{1, k}^{(k-1)}, \ldots, \widetilde{p}_{N, k}^{(k-1)}\right)^{T}$, and $Y^{(k-1)}=\left(y_{1}^{(k-1)}, \ldots, y_{N}^{(k-1)}\right)^{T}$, and $R_{k-1}$ is the $(k-1) \times(k-1)$ upper triangular matrix. Further denote

$$
\begin{array}{r}
a_{j}^{(k)}=\left(\sum_{i=k}^{N}\left(\tilde{p}_{i, j}^{(k-1)}\right)^{2}\right)^{1 / 2} ; \quad b_{j}^{(k)}=\sum_{i=k}^{N} \tilde{p}_{i, j}^{(k-1)} y_{i}^{(k-1)}, \\
j=k, \ldots, M .
\end{array}
$$

Assume that the maximum of $\left(b_{j}^{(k)} / a_{j}^{(k)}\right)^{2}, j=k, \ldots, M$, is achieved at $j=j_{m}$. Then interchange the $j_{m}$ th column of $\tilde{p}_{k}^{(k-1)}$ with the $k$ th column. The procedure is terminated at $M_{s}$ th stage when

$$
1-\sum_{i=1}^{M_{s}} \frac{\left(y_{j}^{\left(M_{s}\right)}\right)^{2}}{\langle Y, Y\rangle} \leq \rho, \quad \text { or } \quad M_{s}=M,
$$

where $\rho(0<\rho \leq 1)$ is a desired tolerance. Using backward substitution, the subset model parameter estimate $\Theta_{s}$ is computed from

$$
R_{M_{s}} \Theta_{s}=\left[y_{1}^{\left(M_{s}\right)} \cdots y_{M_{s}}^{\left(M_{s}\right)}\right]^{T} .
$$

In addition, since the terms of unmeasured disturbance driving force are generally unmeasured, the identification will require an iterative approach. The identification procedures can be clarified as follows.

Step 1. Set the initial sequence $\alpha_{0, t}$ by fitting a linear model or setting the $\alpha_{0, t}$ to zero, and set iteration number $i=1$.

Step 2. Identify the nonlinear model and get the prediction errors or residuals $\xi_{k}^{[i]}, k=1, \ldots N$.

Step 3. If certain identification criteria are achieved, then the program jumps to Step 6. Otherwise, Step 4 is run.

Step 4. Replace the initial sequence by the prediction errors or residuals.

Step 5. Set iteration number $i=i+1$ and return to Step 2 .

Step 6. End of program.

Once the parameters of the closed-loop model are estimated, Monte Carlo (MC) method may be used to compute 
TABLE 1: The obtained model coefficients and minimum variance by linear estimation method.

\begin{tabular}{|c|c|c|c|c|c|c|}
\hline \multirow{2}{*}{ Terms } & \multicolumn{3}{|c|}{$b=3, l=5$} & \multicolumn{3}{|c|}{$b=5, l=3$} \\
\hline & Real values & Estimated values & Variances & Real values & Estimated values & Variances \\
\hline 1 & 1 & 1 & 0 & 1 & 1 & 0 \\
\hline$\psi_{0,1}$ & 1.60 & 1.4977 & 0.0118 & 1.60 & 1.3811 & 0.0172 \\
\hline$\psi_{0,2}$ & 1.76 & 1.4321 & 0.0396 & 1.76 & 1.2671 & 0.0466 \\
\hline$\psi_{0,3}$ & $x$ & $x$ & $x$ & 1.54 & 1.0338 & 0.0801 \\
\hline$\psi_{0,4}$ & $x$ & $x$ & $x$ & 1.05 & 0.6427 & 0.0825 \\
\hline$\sigma_{0}^{2}$ & 0.05 & 0.1208 & 0.0040 & 0.05 & 0.1712 & 0.0230 \\
\hline$\psi_{1,0}$ & $x$ & $x$ & $x$ & $x$ & $x$ & $x$ \\
\hline$\psi_{1,1}$ & $x$ & $x$ & $x$ & $x$ & $x$ & $x$ \\
\hline$\psi_{1,2}$ & $x$ & $x$ & $x$ & $x$ & $x$ & $x$ \\
\hline$\psi_{1,3}$ & $x$ & $x$ & $x$ & 1 & 1.0005 & 0.0234 \\
\hline$\psi_{1,4}$ & $x$ & $x$ & $x$ & 0.3 & 0.2849 & 0.0588 \\
\hline$\sigma_{1}^{2}$ & 0.1 & 0.0997 & $2.1861 e-004$ & 0.1 & 0.0989 & $2.0217 e-004$ \\
\hline$\sigma_{\mathrm{mv}}^{2}$ & 0.3329 & 0.7038 & 0.0919 & 0.6156 & 1.1688 & 0.2958 \\
\hline
\end{tabular}

the variance decomposition. Firstly, two random vectors, $\dot{x}^{(k)}=\left[\dot{x}_{1}^{(k)}, \dot{x}_{2}^{(k)}\right]_{N_{t} \times 1}$ and $\ddot{x}^{(k)}=\left[\ddot{x}_{1}^{(k)}, \ddot{x}_{2}^{(k)}\right]_{N_{t} \times 1}$, are generated, which are two sets of $N_{\mathrm{mc}}$ simulation of multidimensional inputs that have the requisite distribution. $N_{t}$ denotes memory length of the model. Then, the mean and variance of $y_{t+b}$ given the initial condition $I_{0}$ can be calculated by

$$
\begin{aligned}
& \widehat{\bar{y}}_{t+b}\left|I_{0} \cong \frac{1}{N} \sum_{k=1}^{N_{\mathrm{mc}}} f_{2}\left(\dot{x}^{(k)}\right)\right| I_{0} ; \\
& \widehat{V}_{x} \mid I_{0} \cong \frac{1}{N} \sum_{k=1}^{N_{\mathrm{mc}}}\left(f_{2}\left(\dot{x}^{(k)}\right) \mid I_{0}\right)^{2}-\left(\hat{\bar{y}}_{t+b} \mid I_{0}\right)^{2} .
\end{aligned}
$$

The partial variances can be estimated as

$$
\begin{aligned}
& \widehat{V}_{1} \mid I_{0} \cong \frac{1}{N} \sum_{k=1}^{N_{\mathrm{mc}}} f_{2}\left(\dot{x}_{1}^{(k)}, \dot{x}_{2}^{(k)}\right) f_{2}\left(\dot{x}_{1}^{(k)}, \ddot{x}_{2}^{(k)}\right)-\left(\widehat{\bar{y}}_{t+b} \mid I_{0}\right)^{2}, \\
& \widehat{V}_{2} \mid I_{0} \cong \frac{1}{N} \sum_{k=1}^{N_{\mathrm{mc}}} f_{2}\left(\dot{x}_{1}^{(k)}, \dot{x}_{2}^{(k)}\right) f_{2}\left(\ddot{x}_{1}^{(k)}, \dot{x}_{2}^{(k)}\right)-\left(\widehat{\bar{y}}_{t+b} \mid I_{0}\right)^{2}, \\
& \widehat{V}_{12}\left|I_{0} \cong \widehat{V}_{x}\right| I_{0}-\widehat{V}_{1}\left|I_{0}-\widehat{V}_{2}\right| I_{0} .
\end{aligned}
$$

To calculate the $\widehat{V}_{1} \mid I_{0}$ with the different initial conditions, the average of these values can be used as the estimates of $E_{I_{0}}\left[V_{1} \mid I_{0}\right]$, and the performance index of nonlinear feedforward and feedback control system can be obtained.

\section{Simulation Study}

This section presents a simulation experiment to show the effectiveness of the proposed strategy. The model of nonlinear feedforward and feedback control system that we have chosen is expressed as

$$
y_{t}=f\left(u_{t-b}^{*}\right)+D_{0, t}+q^{-3}\left(1-0.6 q^{-1}\right) D_{1, t},
$$

where $f\left(u_{t-b}^{*}\right)$ is process model represented by a nonlinear polynomial:

$$
\begin{aligned}
f\left(u_{t-b}^{*}\right)= & 0.2 u_{t-3}+0.3 u_{t-4}+u_{t-5}+0.8 u_{t-3}^{2} \\
& +0.8 u_{t-3} u_{t-4}-0.7 u_{t-4}^{2}-0.5 u_{t-5}^{2}-0.5 u_{t-3} u_{t-5}
\end{aligned}
$$

The measured and unmeasured disturbances are, respectively, given by

$$
D_{0, t}=\frac{1}{1-1.6 q^{-1}+0.8 q^{-2}} \alpha_{0 . t}, \quad D_{1, t}=\frac{1}{1-0.9 q^{-1}} \alpha_{1, t},
$$

where $\left\{\alpha_{0, t}\right\}$ and $\left\{\alpha_{1, t}\right\}$ are sequences of independent and identically distributed normal variables with mean zero, and the variances are, respectively, 0.05 and 0.1 .

Assume that the process is presently being controlled about a fixed set point by a simple proportional feedforward controller in addition to an integral feedback controller. The manipulated variable is given by

$$
u_{t}=-0.1 D_{1, t}-\frac{0.3-0.2 q^{-1}}{1-q^{-1}} y_{t}
$$

Two closed-loop signal curves of different time-delay conditions $b=3, l=5$ and $b=5, l=3$ are shown in Figure 2 . Then, the traditional linear regression method is applied to estimate the MVPLB for nonlinear forward feedback control system. The estimated values of model parameters and MVPLB are shown in Table 1, where the model orders are $J_{0}=7, J_{1}=7$, and $J_{D}=1$ by applying AIC criterion and the values are calculated by 100 times' statistics. It can be seen that the estimated value of model parameters and MVPLB by traditional linear regression method has larger deviation, which is always larger than the real value. This implies the excessive estimation.

It is necessary to identify the model of closed-loop system to estimate the minimum variance performance index of the 

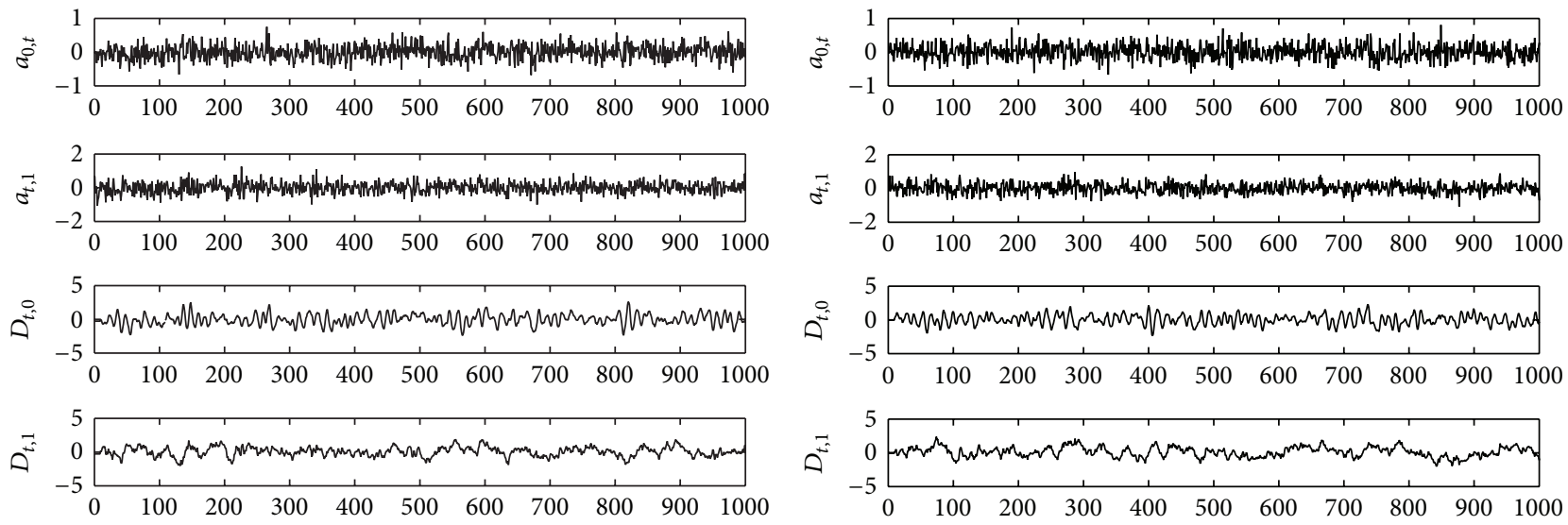

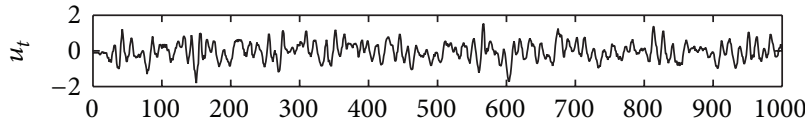
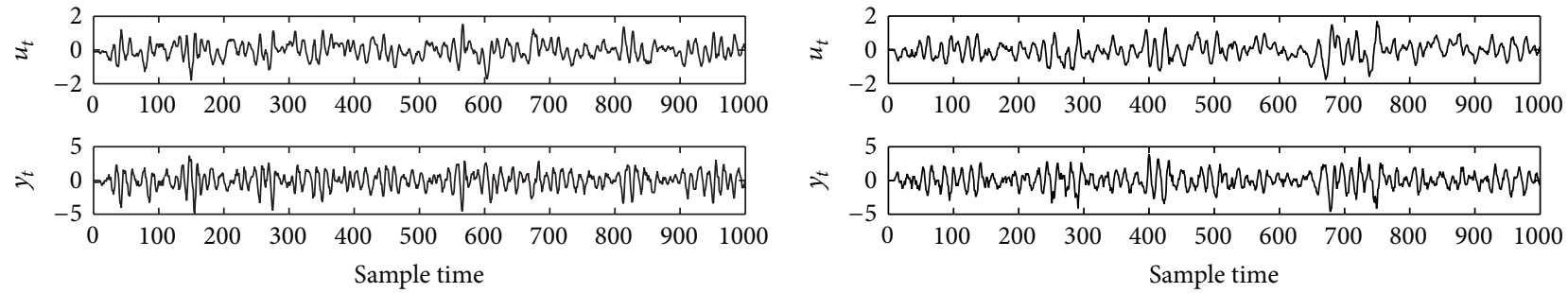

(a)

(b)

FIGURE 2: 1000 samples for the closed-loop nonlinear feedforward and feedback system subjected to measured and unmeasured disturbances. (a) $b=3, l=5$; (b) $b=5, l=3$.

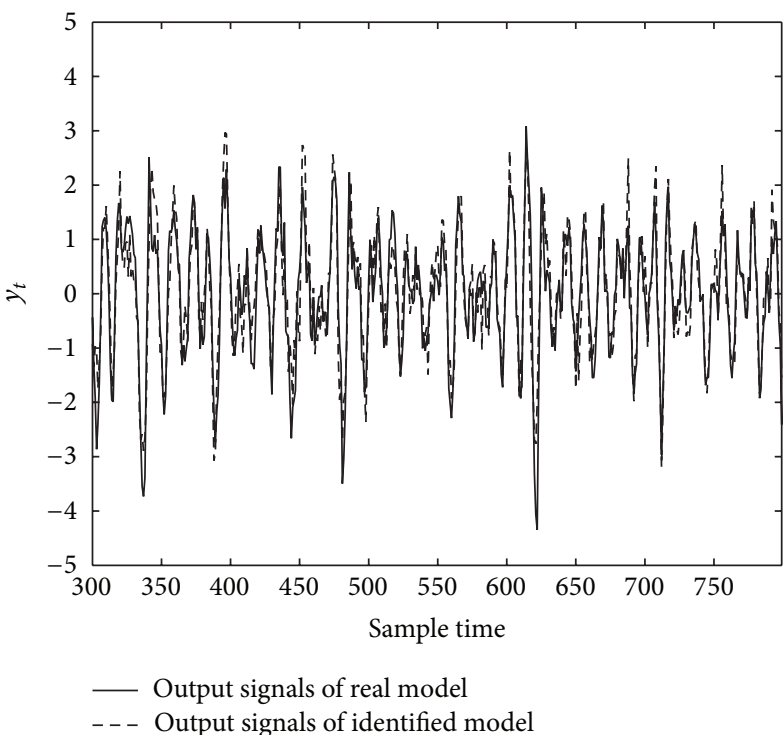

(a)

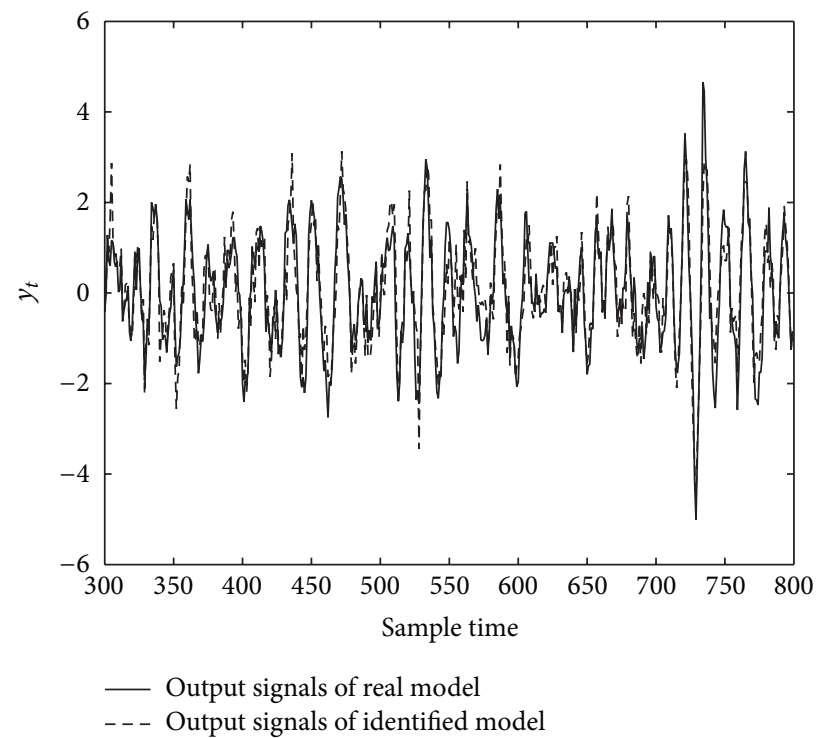

(b)

FIGURE 3: Output signals of identified model comparing with actual model. (a) $b=3, l=5$; (b) $b=5, l=3$.

nonlinear system. First, we collect the disturbance signals which can be measured and then apply the linear regression method to fit the curve to obtain the parameter of the white noise. Furthermore, we use iterative orthogonal least square method to identify the closed-loop model. The comparison for the output signal of identified model and actual model under two different time delays is shown in Figure 3. We can see the identified model can well approximate to the real nonlinear model.
It is noted that the output variance of nonlinear system is also related to the initial value. Thus, to see whether the resulting controller performance based on variance decomposition method includes the influence of the initial value or not, the output variation of closed-loop system during the period $t=1,2, \ldots, 40$ is shown in Figure 4 . It can be seen that when $t>20$, the distribution of the system output tends to be stable; thus we get the conclusion that the output has nothing to do with the initial value. 


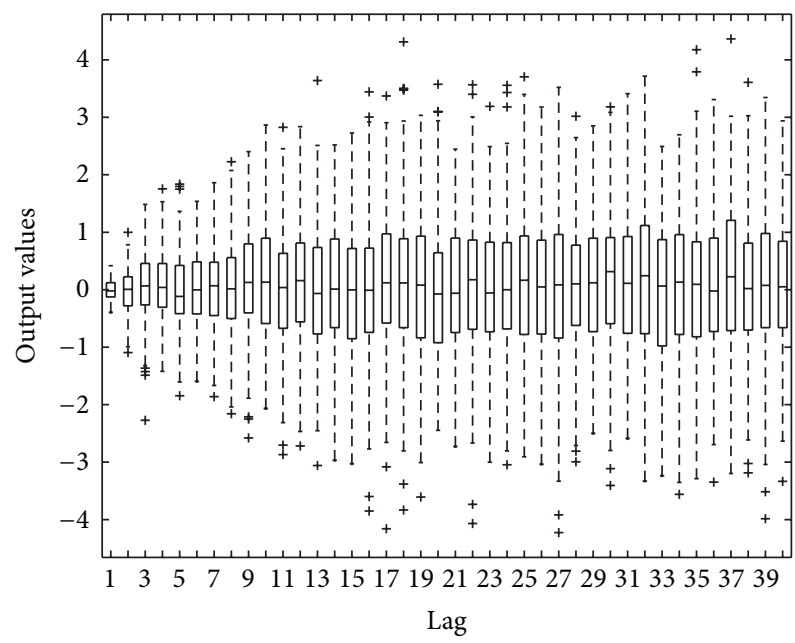

(a)

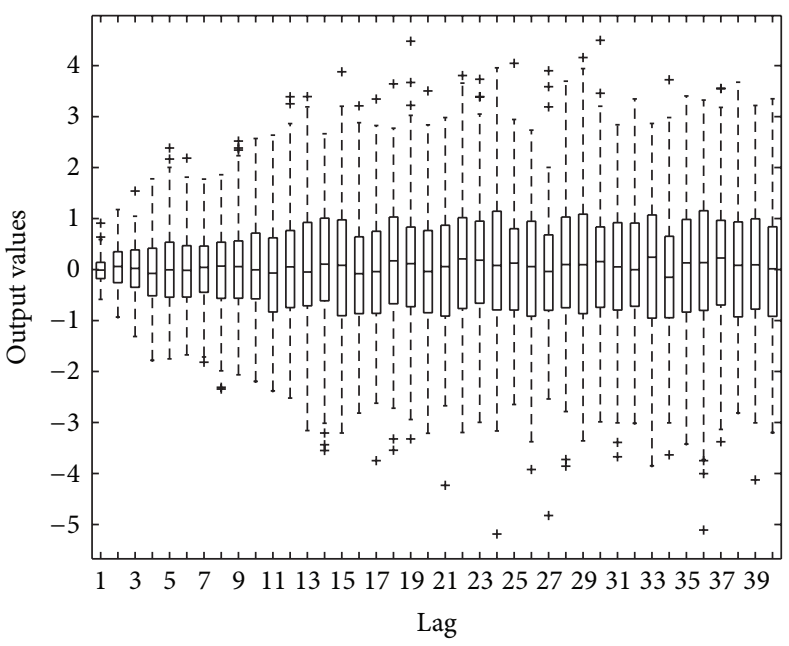

(b)

Figure 4: Box plots for closed-loop system output on memory length $t=1, \ldots, 40$. (a) $b=3, l=5$; (b) $b=5, l=3$.

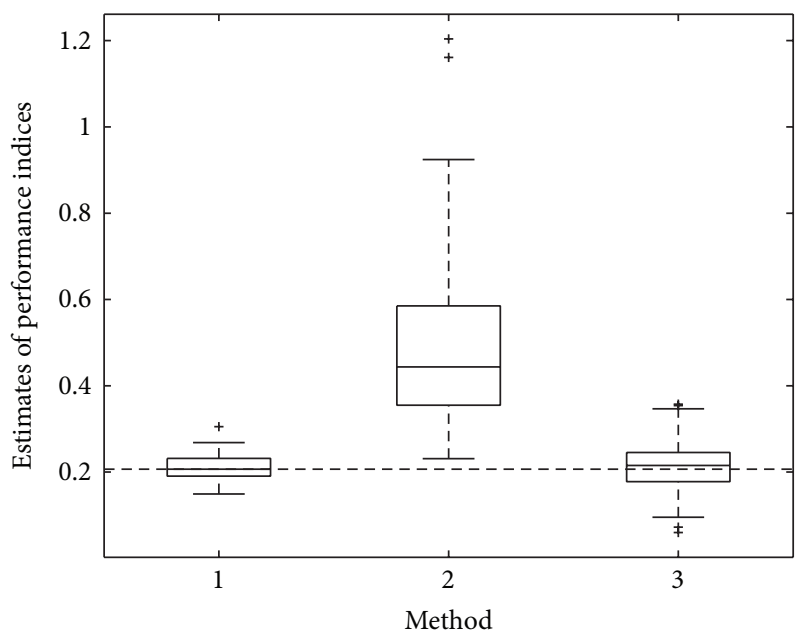

(1) Theoretical performance index

(2) Linear autoregressive method

(3) Proposed method in this paper

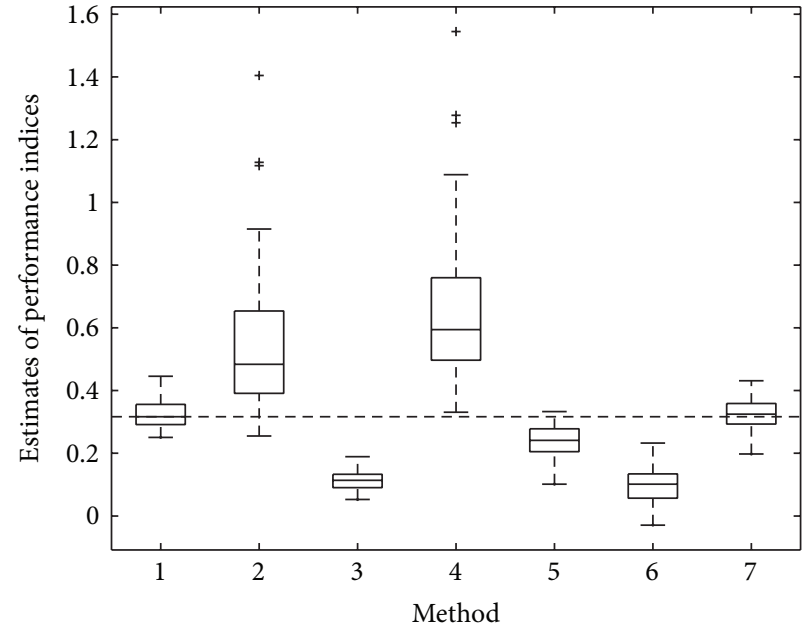

(1) Theoretical performance index

(2) Contribution of unmeasured disturbance with LAR method

(3) Contribution of measured disturbance with LAR method

(4) Performance index with LAR method

(5) Contribution of unmeasured disturbance with proposed method

(6) Contribution of measured disturbance with proposed method

(7) Performance index with proposed method

(b)

(a)

Figure 5: Box plots of the estimates of the minimum variance lower bound for the nonlinear feedforward and feedback control system. (a) $b=3, l=5$; (b) $b=5, l=3$.

Selecting an appropriate memory length of 40 and applying 100 times' Monte Carlo experiments, the box plots of MVPLB estimates with time delay $b=3, l=5$, and $b=5$, $l=3$ by applying variance decomposition method proposed by this paper and traditional linear estimation method can be seen in Figure 5. In Figure 5(a), the first column gives theoretical performance index for nonlinear system with time delay $b=3, l=5$, and the second column and third one, respectively, show the estimates of performance index by traditional linear method and that by the method in this paper. In Figure 5(b), the first column gives theoretical performance index for nonlinear system with time delay $b=5, l=3$, and the fourth column and seventh column, respectively, show the estimates of performance index by traditional linear 
method and that by the method in this paper. The second and third column, respectively, show the contributions of performance index of the unmeasured and measured disturbance signal applying traditional linear method. The fifth and sixth column show the contributions of performance index of the unmeasured and measured disturbance signal applying the method proposed by this paper, respectively. From the plot, we can see that the estimates of performance index using our new method are more close to the theoretical value than that using traditional linear method and get the conclusion that it is effective to estimate the MVPLB of nonlinear forward and feedback system by applying the CPA method based on variance decomposition method.

Remarks. (i) The MVPLB of this nonlinear feedback and forward control system can be decomposed into the best possible bounds for each of the controllers. According to the variance contributions of the unmeasured and measured disturbance, we can confirm the degree of controller performance by the feedback controller and the feedforward controller.

(ii) When the feedforward delay exceeds the feedback delay, there is no error in predicting of the future disturbance by using given information at current time. In such case, the overall MVPLB is only the contribution of unmeasured disturbance. This is the reason why only three columns are included in Figure 5.

(iii) This new nonlinear CPA method requires only observable signals and crude estimates of the process delay and another delay that it takes for a change in measured feedforward variable to begin to affect the output.

(iv) The proposed method needs to estimate the closedloop nonlinear model, and the identification of the closedloop model will directly affect the estimates of the MVPLB.

\section{Conclusions}

The problem of control performance assessment for nonlinear feedforward and feedback system is investigated in this paper. We provide a method based on the variance decomposition to estimate the MVPLB for two classes of nonlinear feedforward and feedback control system. When the time delay of the process and measured disturbance are known, the performance index based on minimum variance benchmark can be estimated by the data from the closedloop system; the simulation shows the effectiveness of the proposed approach. More specifically, the assumption of one measured disturbance is also suitable for the multimeasured disturbance cases; thus the method in this paper can be extended from SISO to MISO.

\section{Conflict of Interests}

The authors declare that there is no conflict of interests regarding the publication of this paper.

\section{Acknowledgments}

This work was supported by the Priority Academic Program Development of Jiangsu Higher Education Institutions and the 111 Project (B12018) and the Basic Research Program of Jiangsu Province of China (Natural Science Foundation) (BK2012111).

\section{References}

[1] T. J. Harris, "Assessment of control loop performance," The Canadian Journal of Chemical Engineering, vol. 67, no. 5, pp. 856-861, 1989.

[2] L. Desborough and T. Harris, "Performance assessment measures for univariate feedback control," The Canadian Journal of Chemical Engineering, vol. 70, no. 6, pp. 1186-1197, 1992.

[3] N. Stanfelj, T. E. Marlin, and J. F. MacGregor, "Monitoring and diagnosing process control performance: the single-loop case," Industrial and Engineering Chemistry Research, vol. 32, no. 2, pp. 301-314, 1993.

[4] L. Desborough and T. Harris, "Performance assessment measures for univariate feedforward/feedback control," The Canadian Journal of Chemical Engineering, vol. 71, no. 4, pp. 605-616, 1993.

[5] T. J. Harris, F. Boudreau, and J. F. MacGregor, "Performance assessment of multivariable feedback controllers," Automatica, vol. 32, no. 11, pp. 1505-1518, 1996.

[6] B. Huang, S. L. Shah, and E. K. Kwok, "Good, bad or optimal? Performance assessment of multivariable processes," Automatica, vol. 33, no. 6, pp. 1175-1183, 1997.

[7] B. Huang, S. L. Shah, and R. Miller, "Feedforward plus feedback controller performance assessment of MIMO systems," IEEE Transactions on Control Systems Technology, vol. 8, no. 3, pp. $580-587,2000$.

[8] S. J. Qin, "Control performance monitoring-a review and assessment," Computers and Chemical Engineering, vol. 23, no. 2, pp. 173-186, 1998.

[9] B. Huang and S. L. Shah, Performance Assessment of Control Loops: Theory and Applications, Springer, London, UK, 1999.

[10] M. Jelali, "An overview of control performance assessment technology and industrial applications," Control Engineering Practice, vol. 14, no. 5, pp. 441-466, 2006.

[11] B. Srinivasan, T. Spinner, and R. Rengaswamy, "Control loop performance assessment using detrended fluctuation analysis (DFA)," Automatica, vol. 48, no. 7, pp. 1359-1363, 2012.

[12] R. Gonzalez and B. Huang, "Control loop diagnosis with ambiguous historical operating modes: part 1. A proportional parametrization approach Original Research Article," Journal of Process Control, vol. 23, no. 4, pp. 585-597, 2013.

[13] R. Gonzalez and B. Huang, "Control loop diagnosis with ambiguous historical operating modes: part 2, information synthesis based on proportional parametrization," Journal of Process Control, vol. 23, no. 10, pp. 1441-1454, 2013.

[14] S. Liu, J. F. Fiu, Y. P. Feng et al., "Performance assessment of decentralized control systems: an iterative approach," Control Engineering Practice, vol. 22, pp. 252-263, 2014.

[15] T. J. Harris and W. Yu, "Controller assessment for a class of nonlinear systems," Journal of Process Control, vol. 17, no. 7, pp. 607619, 2007

[16] W. Yu, D. I. Wilson, and B. R. Young, "Nonlinear control performance assessment in the presence of valve stiction," Journal of Process Control, vol. 20, no. 6, pp. 754-761, 2010.

[17] W. Yu, D. I. Wilson, and B. R. Young, "Control performance assessment for nonlinear systems," Journal of Process Control, vol. 20, no. 10, pp. 1235-1242, 2010. 
[18] Z. Zhang, On Performance assessment for nonlinear control systems based on mimimum variance benchmark [Ph.D. thesis], Shanghai Jiao Tong University, Shanghai, China, 2013.

[19] W. Yu, D. I. Wilson, and B. R. Young, "Control performance assessment for a class of nonlinear multivariable systems," Computer Aided Chemical Engineering, vol. 30, pp. 962-966, 2012.

[20] W. Yu, D. Wilson, B. Young, and T. Harris, "Variance decomposition of nonlinear systems," in Proceedings of the IEEE International Conference on Control and Automation (ICCA '09), pp. 738-744, Christchurch, New Zealand, December 2009.

[21] G. E. B. Archer, A. Saltelli, and I. M. Sobol, "Sensitivity measures, anova-like techniques and the use of bootstrap," Journal of Statistical Computation and Simulation, vol. 58, no. 2, pp. 99-120, 1997.

[22] S. Chen, S. A. Billings, and W. Luo, "Orthogonal least squares methods and their application to nonlinear system identification," International Journal of Control, vol. 50, no. 5, pp. 18731896, 1989. 


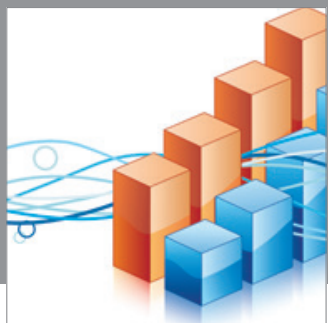

Advances in

Operations Research

mansans

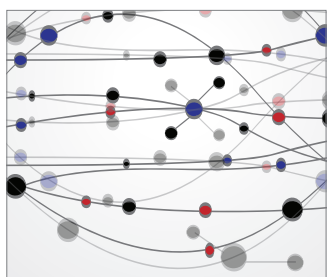

The Scientific World Journal
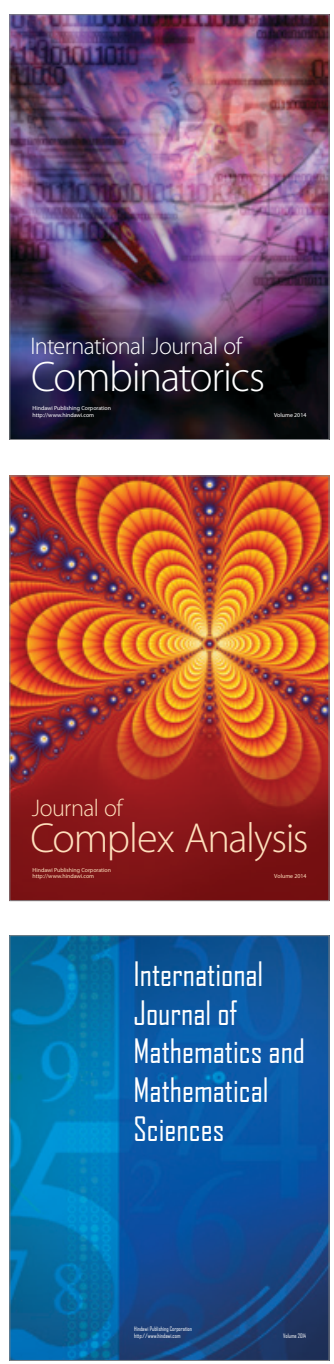
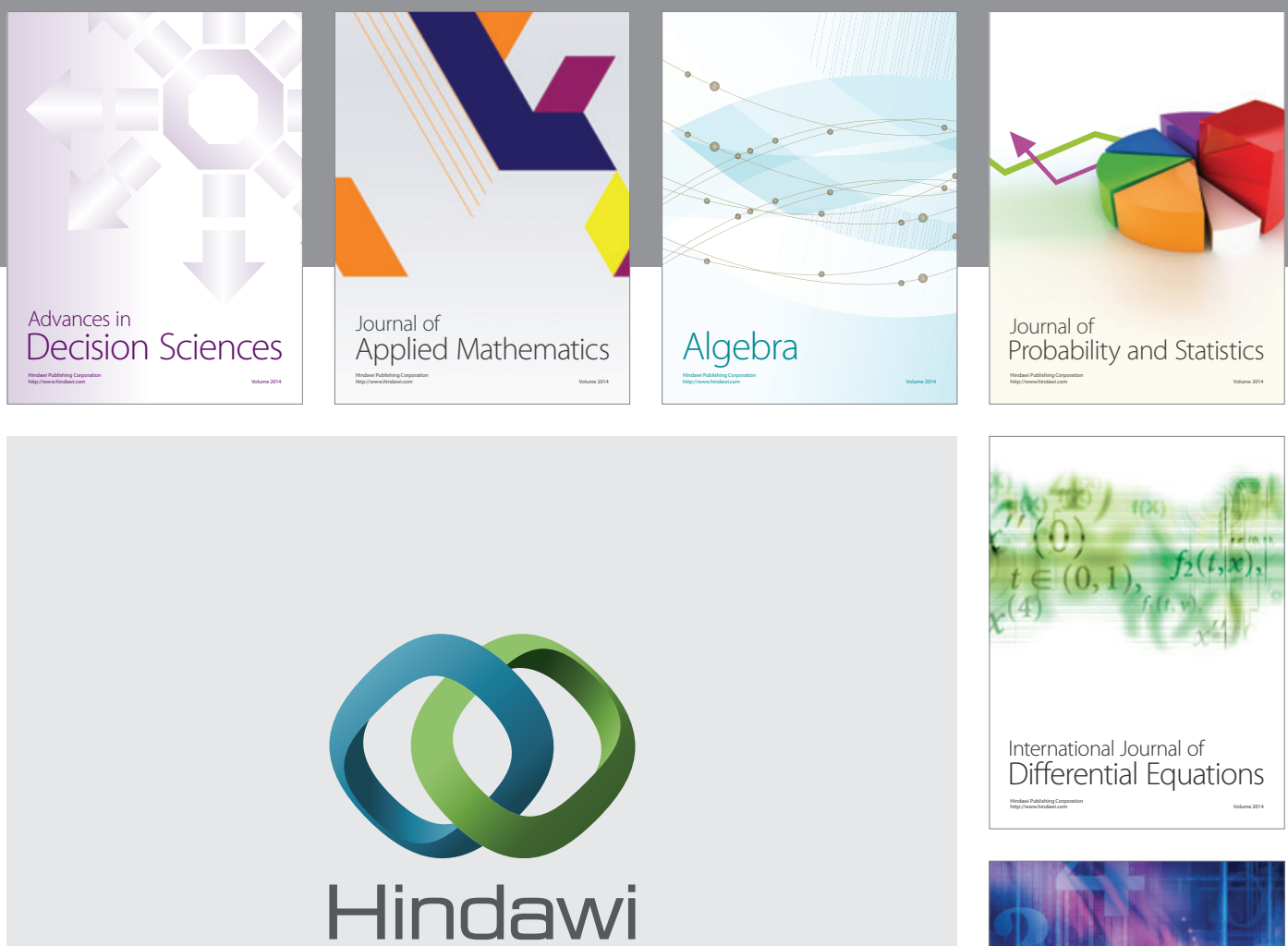

Submit your manuscripts at http://www.hindawi.com
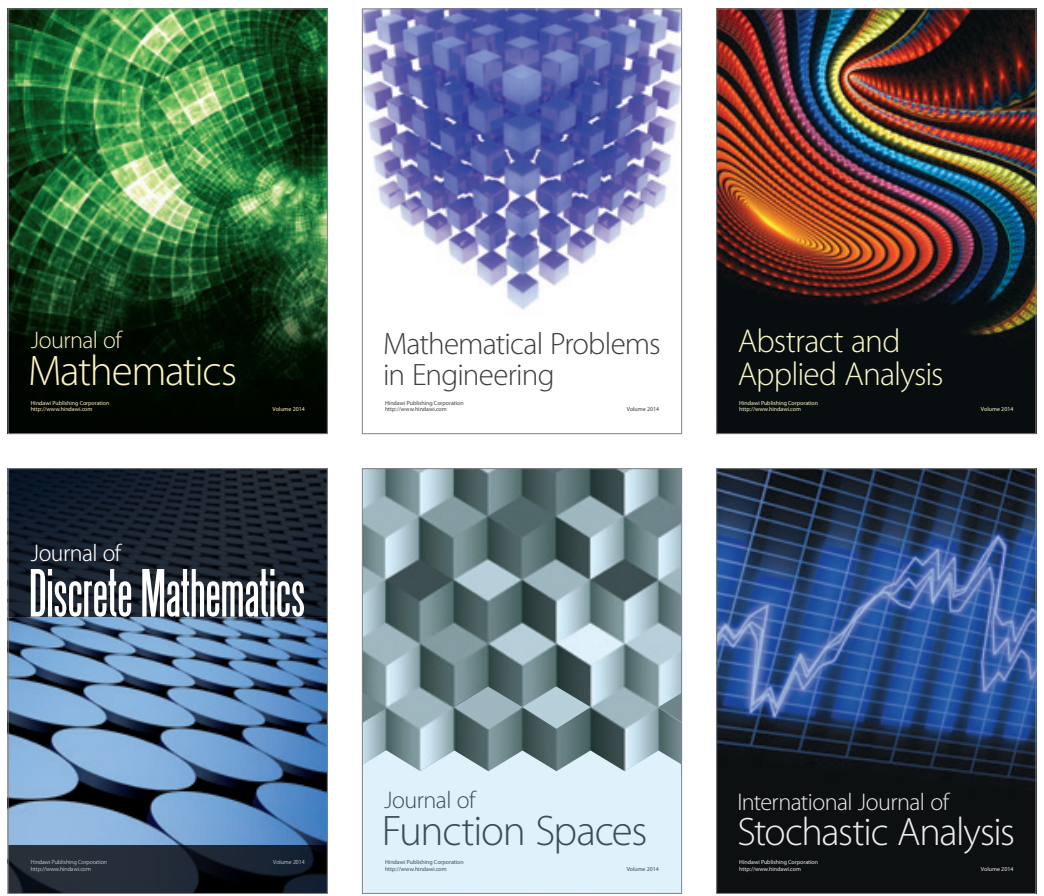

Journal of

Function Spaces

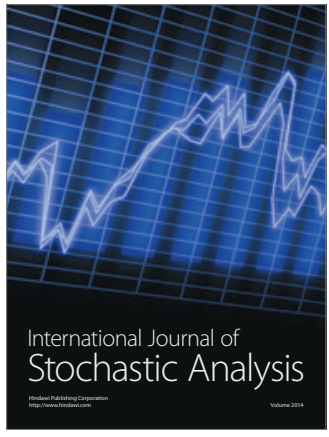

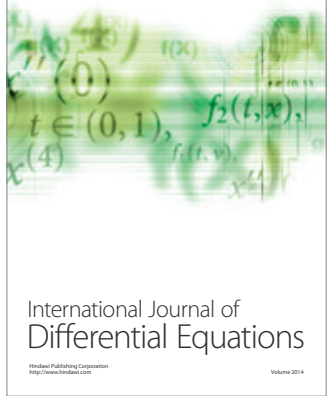
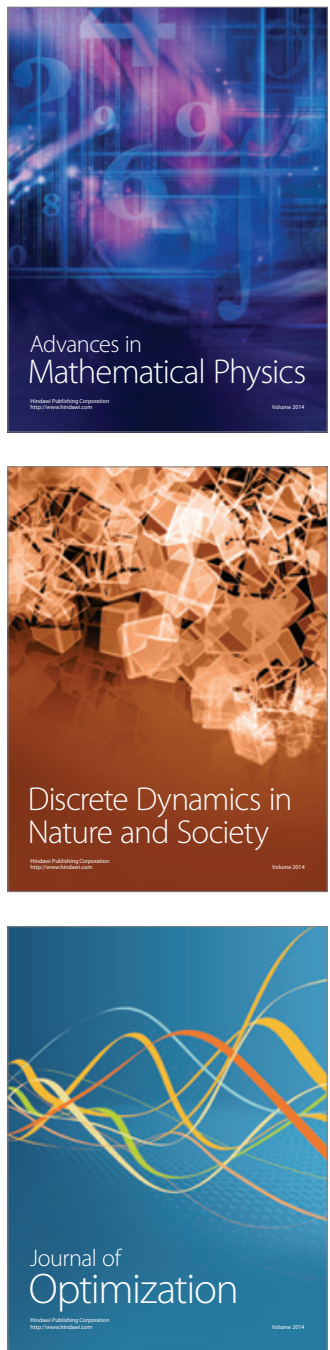